\title{
On the extrapolation of acoustic waves from flow simulations with vortical outflow
}

\author{
M. C. M. Wright \\ C. L. Morfey \\ Institute of Sound \& Vibration Research \\ University of Southampton, UK \\ email: mcmw@isvr.soton.ac.uk
}

\begin{abstract}
Feri Farassat was one of the pioneers of the use of the Ffowcs Williams \& Hawkings formulation of Lighthill's acoustic analogy as a way to extrapolate radiated waves from simulations of unsteady flows. Current computational limits mean that volume source terms are often neglected, causing inaccurate acoustical predictions when entropy fluctuations or vorticity pass across the extrapolation surface. The derivation of the Ffowcs Williams-Hawkings equation is modified to allow the equivalent surface sources to be distributed over a transition layer of finite thickness rather than being confined to a single layer, in order to reduce the effect of vorticity exiting the computational domain.
\end{abstract}

\section{Introduction}

Aeroacoustics is often considered to have had two golden ages, the first inaugurated by Lighthill's 1952 formulation of his acoustic analogy, and the second arising with the possibility of computing sound fields in the course of unsteady flow simulations. Feri Farassat made many contributions to the subject, but one of the most important was the application of a technique from the first golden age, the Ffowcs 
Williams-Hawkings (FWH) form of the acoustic analogy, to the problems of the second. Di Francescantonio showed in 1997 that an FWH surface could be used as an extrapolation surface at an arbitrary location instead of at the surface of a solid body (although Ffowcs Williams and Hawkings 1969 had presciently allowed the possibility of flow through the surface, and this was maintained in Dowling \& Ffowcs Williams 1983) and other aeroacoustics researchers were close behind. But it was Brentner and Farassat's 1998 comparison of the FWH and Kirchhoff formulations that established the FWH surface as the method of choice for extrapolating radiated sound fields from unsteady flow simulations.

Since then it has become standard practice to neglect the contribution from distributed volume sources so that only sources confined to the extrapolation surface contribute to the sound field. For the density form of the FWH equation this corresponds to neglecting quadrupole sources. This can be justified both by the placement of the extrapolation surface outside the region in which these sources are strong, and for low-Mach-number problems by the relative scaling of dipole and quadrupole terms. The benefit of neglecting volume sources is computational; less data needs to be stored and fewer source calculations need to be made. For jet modelling, however, it may not be practical to close the extrapolation surface so far downstream that vorticity and entropy fluctuations have decayed. In this case neglecting the volume sources in the FWH formulation can produce considerable spurious noise; spurious because the convection of entropy fluctuations and/or vorticity should be silent. The problem arises because when a 'hot spot' or an eddy passes through the extrapolation surface it generates both surface and volume sources in the FWH formulation that cancel each other out. The spurious noise is due to the absence of volume sources to cancel the surface sources.

Wang et al. (1996) proposed the first practical solution to the problem of spurious sound generation by subsonic eddy structures as they convect out of the simulation domain, as outlined in section 2.1 below. Shur et al. (2005) encountered both these issues with hot jets and proposed solutions: changing to a pressure-like variable for 
the entropy fluctuations, and averaging over two or more closely-spaced FWH surfaces for the vorticity. The effectiveness of this strategy, compared to a single surface, was verified by Spalart \& Shur (2009) and by Mendez et al. (2013).

In Morfey \& Wright (2007) we derived an acoustic analogy in terms of pressurelike variables (related to that used in Morfey 1973) and showed that additional volume sources, not necessarily quadrupoles, arise. We deduced that this formulation should produce less spurious entropy noise despite the neglect of all volume sources, as had already been demonstrated numerically by Shur et al. (2005). In this article we shall derive a form of the acoustic analogy equation in which the zero-thickness source layer of the FWH formulation (Fig. 1, bottom-left) is replaced by similar sources distributed over a finite-thickness transition region (Fig. 1, bottom-centre). The purpose of this reformulation is that when an eddy passes through the transition region its contribution to the sound field should fade out smoothly, thus minimizing the spurious noise. We then show that the same general form can lead to an acoustic analogy with multiple nested extrapolation surfaces (Fig. 1, bottom-right) of which the averaging procedure of Shur et al. (2005) is a particular case. Whether an optimized version of such a procedure would be better or worse than the alternative of adding a correction term to account for vorticity convection across the boundary, as originally proposed by Wang et al. (1996), remains to be determined by numerical experiment.

\section{Altenative approaches to the outflow boundary problem}

\subsection{The frozen-eddy correction procedure}

In order to illustrate the problem, Wang et al. (1996) used a 2D incompressible DNS simulation of unsteady vortex shedding by an airfoil at incidence. The volume and surface integrals required by Curle's (1955) extension of Lighthill's acoustic analogy were evaluated using data extracted from the simulation. Because of the finite computational box, volume quadrupole sources associated with the wake 
further downstream were not captured, leading to spurious additional sound radiation. The effect is exactly the same as would be produced by a FWH permeable-surface calculation that used DNS data on a control surface $\mathcal{S}$, with $\mathcal{S}$ coinciding with the box boundaries.

In both cases, neglect of quadrupoles in the wake downstream of $\mathcal{S}$ is responsible for significant errors in the sound field. Wang et al. (1996) showed that in their example, such errors could be greatly reduced by introducing a correction term based on the assumption of frozen eddy convection across the exit boundary.

The correction-term idea of Wang et al. has been extended in subsequent studies, notably in Avital et al. (1999) and Ikeda et al. (2013). Whereas the methods of Wang et al. (1996) and Avital et al. (1999) are based on the acoustic analogy volume-source representation, with data from a finite simulation domain providing volume source terms as input to a far-field radiation integral, more recent work exemplified by Ikeda et al. (2013) starts from the FWH porous-surface formulation. We emphasise that whichever approach is used, the same truncation error will occur in the absence of any correction procedure: the error arises from the omission of acoustic-analogy volume sources that lie outside the integration domain $\mathcal{D}$ or control surface $\mathcal{S}$.

\subsection{Smoothing of the spatial integration window}

Obrist \& Kleiser (2007) pointed out, in the context of the volume-source representation, that sharp spatial truncation of the physical source domain by a rectangular, or top-hat, 3D window is a source of spectral leakage in the wavenumber domain. The resultant leakage of equivalent sources to low wavenumbers (specifically, to wavenumbers in the acoustic range) has the effect of maximizing the truncation error discussed in section 2.1. They therefore proposed using a smoothly-tapered spatial window in order to evaluate the radiation integral. For a subsonic turbulent-jet simulation similar to that of Freund (2001) they showed that window tapering, particularly at the downstream domain boundary, produced significant beneficial effects.

As Obrist \& Kleiser (2007) observed, use of the FWH permeable-surface 
formulation is exactly equivalent to use of a truncated volume-source integral, provided the control surface $\mathcal{S}$ in the FWH formulation coincides with the top-hat spatial window used for the volume integral. In both cases the error is due to omission of a volume-source integral over the domain exterior to $\mathcal{S}$. In what follows, we shall use a similar smoothed-window approach to produce a modified version of the FWH surface-source formulation.

Our aim is to retain the versatility of the FWH approach—in particular, its need to apply the acoustic analogy only in a domain exterior to the main source region-while minimizing the spectral leakage problem highlighted by Obrist \& Kleiser (2007), and at the same time avoiding the frozen-eddy hypothesis described in section 2.1.

\section{Derivation}

\subsection{FWH form of windowed acoustic analogy}

The FWH analogy arises, as does Lighthill's, from the equations of conservation of mass and momentum:

$$
\frac{\partial}{\partial t}\left(\rho-\rho_{0}\right)+\frac{\partial}{\partial x_{i}}\left(\rho u_{i}\right)=0, \quad \frac{\partial}{\partial t}\left(\rho u_{i}\right)+\frac{\partial}{\partial x_{j}}\left(\rho u_{i} u_{j}+p_{i j}\right)=0,
$$

where $\rho$ is the density, $\rho_{0}$ is its quiescent value in the acoustic far field and $p_{i j}$ is the stress tensor due to pressure and viscosity (body forces have been ignored for simplicity). In each case the quantity $\rho u_{i}$ is eliminated and the result manipulated into the form of an inhomogeneous wave equation. If the wavespeed for that wave equation is chosen to match the acoustic wavespeed in a region of fluid into which sound propagates according to that wave equation, then the source terms can be used to infer the sound field radiated by a region of unsteady flow embedded in that region.

The FWH equation generalizes Lighthill's by multiplying the conservation equations (1) with a generalized function that sets them to zero in the flow region but leaves them unchanged outside that domain, namely $\mathrm{H}\left(f\left(x_{i}, t\right)\right)$, a Heaviside function of an indicator function $f\left(x_{i}, t\right)$ that is positive in some region $\mathcal{V}$ and negative in 


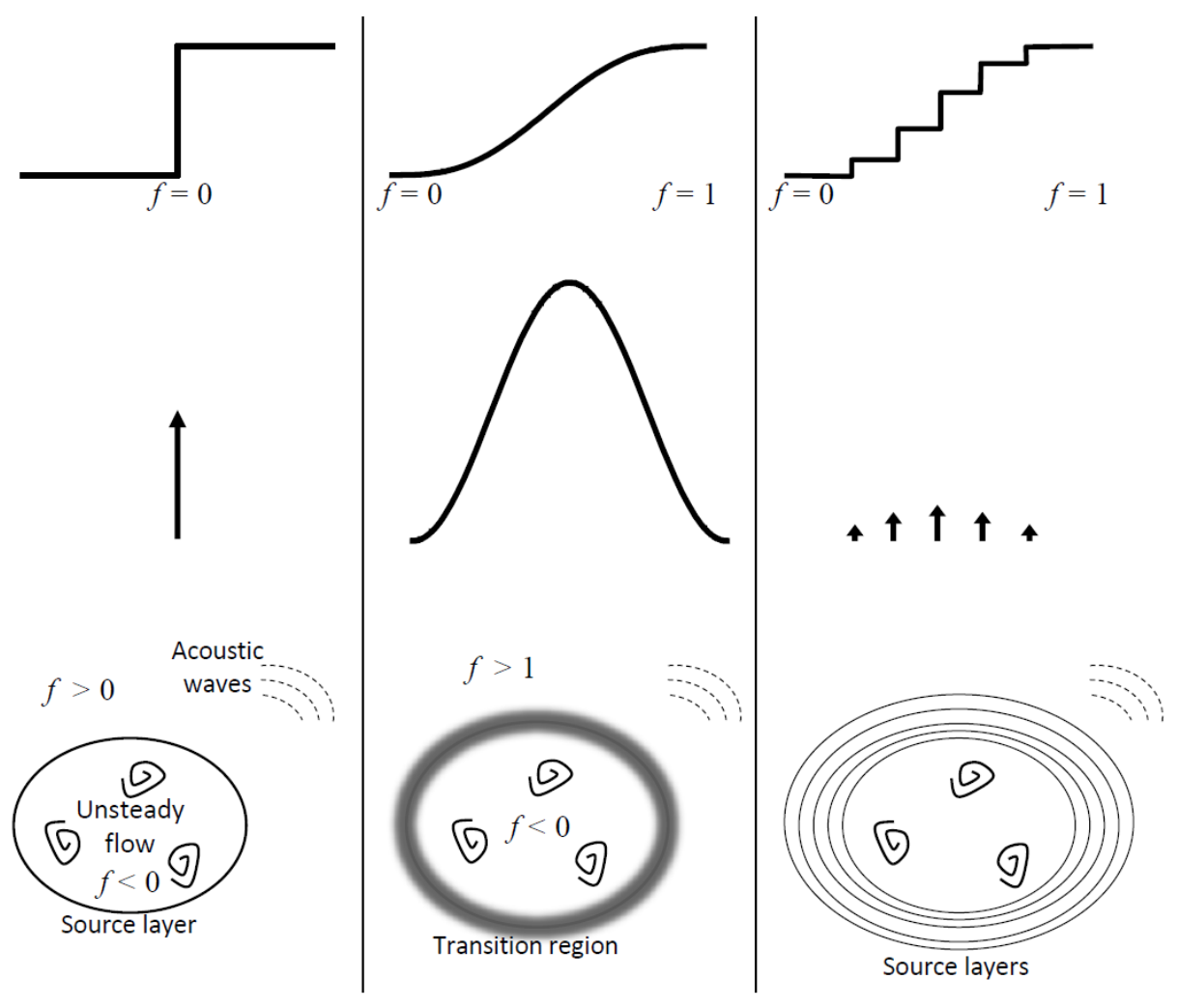

Figure 1: Sketches of $W(f)$ (top row), $W^{\prime}(f)$ (middle) and the resulting wave extrapolation configuration (bottom) for the three formulations described: Ffowcs Williams-Hawkings ( $\S 3.3 .1$, left), smooth transition zone ( $\S 3.3 .2$, centre) and stepped transition zone (§3.3.3, right). Vertical arrows indicate Dirac delta functions. Note that for the stepped formulation the surfaces need not be concentric; their spacing depends on $f$. 
its complement $\mathcal{V}^{\prime}$ which it meets at surface $\mathcal{S}$. The process can be thought of as windowing the domain on $\mathcal{V}$. The windowed equations are then manipulated into the form of a wave equation in a windowed variable. Source terms now arise not only from eliminating $\rho u_{i}$ as before, but also from replacing windowed derivatives by derivatives of windowed quantities.

The terminology of windowing is borrowed from signal processing where it is well-known that multiplying a time-domain signal by a window function affects the spectrum of the signal; specifically it convolves the Fourier transform of the unwindowed function with that of the window, and for that reason smooth window functions are often preferred to those based on Heaviside functions. Obrist and Kleiser (2007) used this reasoning to propose that when using Lighthill's equation to predict sound from a region of unsteady flow it would be preferable to use a smooth spatial window rather than to simply truncate it. In what follows we derive a version of the FWH equation that uses an arbitrary window function $W\left(f\left(x_{i}, t\right)\right)$ in place of $\mathrm{H}\left(f\left(x_{i}, t\right)\right)$. If this window function is smooth then the sources that were restricted to the surface $\mathcal{S}$ in the usual formulation will be distributed over a transition zone, whose shape depends on both $W$ and $f$.

The term contour is used below to refer to a three-dimensional level set. It is assumed that the acoustic region into which waves are to be extrapolated is unbounded and surrounds the region of unsteady flow that generates the sound; if this is not the case the terms interior and exterior can be interchanged. The $f$ contours that define the transition zone can move but will here be limited to rigid translation, for use in applications where the unsteady fluid motion is embedded in a uniform flow.

\subsection{General form of windowed acoustic analogy}

In the standard FWH derivation the indicator function $f\left(x_{i}, t\right)$ is only required to be negative in the interior region, positive in the exterior region and zero on a smooth contour that separates them. For a distributed source region we shall require $f$ to be negative in the interior region, increase in value with finite gradient from $\alpha$ to $\beta$ (with 
$\alpha \leq 0$ and $\beta>0$ ) moving outward through a transition region, and be greater than $\beta$ in the exterior region. The transition region should separate the interior and exterior regions. We also require the spatial contours of $f$ to be smooth and simply connected ${ }^{1}$. The window function should satisfy

$$
W(f)= \begin{cases}0 & f<\alpha \\ \text { monotonically non-decreasing } & \alpha<f<\beta \\ 1 & f>\beta\end{cases}
$$

This differs from window functions that are commonly used in spectral analysis, which are usually zero outside some finite range. In fact, the derivative of $W$ takes the form of such a window. Without loss of generality we can set $\alpha=0$ and $\beta=1$ since $f$ can be adjusted to move the locations of the contours $f=0,1$.

The windowed conservation equations are

$$
\begin{gathered}
W(f) \frac{\partial}{\partial t}\left(\rho-\rho_{0}\right)+W(f) \frac{\partial}{\partial x_{i}}\left(\rho u_{i}\right)=0, \\
W(f) \frac{\partial}{\partial t}\left(\rho u_{i}\right)+W(f) \frac{\partial}{\partial x_{j}}\left(\rho u_{i} u_{j}+p_{i j}\right)=0 .
\end{gathered}
$$

These can be rewritten with the help of the following identities, obtained from the chain rule,

$$
\begin{gathered}
W(f) \frac{\partial \xi}{\partial t}=\frac{\partial}{\partial t}[\xi W(f)]-\xi \frac{\partial f}{\partial t} W^{\prime}(f), \\
W(f) \frac{\partial \xi}{\partial x_{i}}=\frac{\partial}{\partial x_{i}}[\xi W(f)]-\xi \frac{\partial f}{\partial x_{i}} W^{\prime}(f),
\end{gathered}
$$

which hold for any $\xi\left(x_{i}, t\right)$ and $f\left(x_{i}, t\right)$, to give

$$
\begin{gathered}
\frac{\partial}{\partial t}\left[\left(\rho-\rho_{0}\right) W(f)\right]+\frac{\partial}{\partial x_{i}}\left[\rho u_{i} W(f)\right]=\left(\rho-\rho_{0}\right) \frac{\partial f}{\partial t} W^{\prime}(f)+\rho u_{i} \frac{\partial f}{\partial x_{i}} W^{\prime}(f), \quad(7) \\
\frac{\partial}{\partial t}\left[\rho u_{i} W(f)\right]+\frac{\partial}{\partial x_{j}}\left[\left(\rho u_{i} u_{j}+p_{i j}\right) W(f)\right]=\rho u_{i} \frac{\partial f}{\partial t} W^{\prime}(f)+\left(\rho u_{i} u_{j}+p_{i j}\right) \frac{\partial f}{\partial x_{j}} W^{\prime}(f) .
\end{gathered}
$$

\footnotetext{
${ }^{1}$ This condition can be relaxed when, for example, there are multiple disjoint regions of soundgenerating flow, but is imposed for simplicity.
} 
Subtracting the divergence of the second from the time-derivative of the first gives

$$
\begin{aligned}
\frac{\partial^{2}}{\partial t^{2}}\left[\left(\rho-\rho_{0}\right) W(f)\right]= & \frac{\partial}{\partial t}\left[\left(\rho-\rho_{0}\right) \frac{\partial f}{\partial t} W^{\prime}(f)\right]+\frac{\partial}{\partial t}\left[\rho u_{i} \frac{\partial f}{\partial x_{i}} W^{\prime}(f)\right] \\
& -\frac{\partial}{\partial x_{i}}\left[\rho u_{i} \frac{\partial f}{\partial t} W^{\prime}(f)\right]-\frac{\partial}{\partial x_{i}}\left[\left(\rho u_{i} u_{j}+p_{i j}\right) \frac{\partial f}{\partial x_{i}} W^{\prime}(f)\right] \\
& -\frac{\partial^{2}}{\partial x_{i} \partial x_{j}}\left[\left(\rho u_{i} u_{j}+p_{i j}\right) W(f)\right] .
\end{aligned}
$$

The right hand side can be simplified by recognising that the contours of $f$ at $x_{i}$ and $t$ will have normal velocity $v_{i}\left(x_{j}, t\right)$ satisfying

$$
\frac{\partial f}{\partial t}+v_{i} \frac{\partial f}{\partial x_{i}}=0
$$

or equivalently

$$
\frac{\mathrm{D} f}{\mathrm{D} t}=\left(u_{i}-v_{i}\right) \frac{\partial f}{\partial x_{i}}
$$

where $u_{i}-v_{i}$ is the velocity with which fluid crosses a contour of $f$. Therefore

$$
\begin{aligned}
\frac{\partial^{2}}{\partial t^{2}}\left[\left(\rho-\rho_{0}\right) W(f)\right]= & \frac{\partial}{\partial t}\left\{\left[\rho u_{i}-\left(\rho-\rho_{0}\right) v_{i}\right] \frac{\partial f}{\partial x_{i}} W^{\prime}(f)\right\} \\
& -\frac{\partial}{\partial x_{i}}\left\{\left[\rho u_{i}\left(u_{j}-v_{j}\right)+p_{i j}\right] \frac{\partial f}{\partial x_{j}} W^{\prime}(f)\right\} \\
& +\frac{\partial^{2}}{\partial x_{i} \partial x_{j}}\left[\left(\rho u_{i} u_{j}+p_{i j}\right) W(f)\right] .
\end{aligned}
$$

which can be used to form an acoustic analogy

$$
\begin{aligned}
\left(\frac{\partial^{2}}{\partial t^{2}}-\nabla^{2}\right)\left[c_{0}^{2}\left(\rho-\rho_{0}\right) W(f)\right]= & \frac{\partial}{\partial t}\left\{\left[\rho u_{i}-\left(\rho-\rho_{0}\right) v_{i}\right] \frac{\partial f}{\partial x_{i}} W^{\prime}(f)\right\} \\
& -\frac{\partial}{\partial x_{i}}\left\{\left[\rho u_{i}\left(u_{j}-v_{j}\right)+p_{i j}\right] \frac{\partial f}{\partial x_{j}} W^{\prime}(f)\right\} \\
& +\frac{\partial^{2}}{\partial x_{i} \partial x_{j}}\left[T_{i j} W(f)\right]
\end{aligned}
$$

where

$$
T_{i j}=\rho u_{i} u_{j}+p_{i j}-c_{0}^{2}\left(\rho-\rho_{0}\right) \delta_{i j},
$$

is the Lighthill stress tensor. The use of Green's functions to solve such equations is discussed by Morfey et al. (2011). 


\subsection{Particular forms of windowed acoustic analogy}

By choosing a specific form for $W(f)$, such as those sketched in the top row of Fig. 1, a particular form of the acoustic analogy will be generated, whose suitability for wave extrapolation can be considered.

\subsubsection{Heaviside function}

If we set $W(f)=\mathrm{H}(f)$, and hence $W^{\prime}(f)=\delta(f)$, and require $\left|\partial f / \partial x_{i}\right|=1$ on $\mathcal{S}$ then $\partial f / \partial x_{i}=\hat{n}_{i}$ becomes the unit normal to $\mathcal{S}$ and we recover the standard FWH equations ${ }^{2}$.

The Ffowcs Williams-Hawkings formulation (and its generalization in Morfey \& Wright 2007) replaces information in an interior region $f<0$ with a layer of monopoles and dipoles on the surface $f=0$ and a distribution of exterior volume sources in the exterior region $f>0$.

\subsubsection{Smooth function}

Setting, for example,

$$
W(f)=f-\frac{\sin (2 \pi f)}{2 \pi}
$$

gives

$$
W^{\prime}(f)=1-\cos (2 \pi f),
$$

which is a Hann function similar to that used by Obrist \& Kleiser (2007), the discrete form of which is widely used in spectral analysis. It has the advantage that $W^{\prime}(f)$

\footnotetext{
${ }^{2}$ It is instructive for the subsequent version to consider the result of instead having, say, $\left|\partial f / \partial x_{i}\right|=2$ on $\mathcal{S}$. Since $f$ is independent of any physical quantities the resulting sources must be independent of the choice of $f$. This can be seen to be the case by recalling that if a function $q(x)$ has $q\left(x_{0}\right)=0$ and no other zeros then $\delta(q)=\left(1 /\left|q^{\prime}\left(x_{0}\right)\right|\right) \delta(x)$, so that setting $|\nabla f|=2$ on $\mathcal{S}$ would double the effect of $\partial f / \partial x_{i}$ and halve the contribution from $\delta(f)$, leaving the final result unchanged as required. The simplification afforded by choosing $\left|\partial f / \partial x_{i}\right|=1$ on $\mathcal{S}$ was first recognised by Farassat (Brentner, personal communication) and first used in Farassat \& Myers (1987); it is now the standard procedure for this derivation.
} 
is zero at $f=0,1$. Substituting these into Eq. (13) generates an acoustic analogy wherein the equivalent sources consist of a finite-thickness layer confined between the surfaces $f=0$ and $f=1$, whose thickness may vary spatially according to the choice of $f$. The resulting inhomogeneous wave equation differs from that of Obrist \& Kleiser; their formulation evaluates the quadrupoles $T_{i j}$ throughout the interior region and truncates them gradually rather than abruptly. The present formulation replaces information from the interior region with a distribution of monopoles and dipoles in the intermediate region $0<f<1$, together with exterior volume sources ${ }^{3}$ in the intermediate and exterior regions $f>0$. Obrist \& Kleiser also proposed an extension of their formulation to the FWH analogy, but for spectral reasons rather than for reduction of spurious vortex noise.

If all terms are retained then this formulation should give the same result as the Ffowcs Williams-Hawkings step-function formulation. Given a sufficiently extensive flow simulation the interior region could be made large enough that the field outside it would be entirely acoustic. In this case the two formulations would still be equivalent even after neglecting exterior volume sources, since the latter would have zero strength outside the interior region. In practice, however, it will often be necessary to extrapolate from a region whose exterior is not entirely acoustic, and we conjecture that the formulation obtained with the form of $W(f)$ given above will reduce the effect of vorticity whose length scale is smaller than the width of the intermediate region, when exterior volume sources are ignored. The advantage of a smooth function over a merely continuous one, such as $W(f)=f$ over $0<f<1$, is that any spurious vorticity contribution will be introduced more gradually. The optimum shape for $W(f)$ will depend on the statistical structure of the vorticity moving through the transition zone.

As in the FWH equation above, the surface sources in (13) involve the gradient of $f$. In many FWH computations the extrapolation surface has sharp corners where the

\footnotetext{
${ }^{3}$ With density as the wave variable these will be the quadrupoles $T_{i j}$, but if a pressure-like variable is used instead, as in Morfey \& Wright (2007), then additional exterior volume sources arise.
} 
gradient is undefined, although such surfaces were excluded from consideration by Ffowcs Williams \& Hawkings (1969). Farassat \& Myers (1990) analysed the contribution from such cusps arising from the $T_{i j}$ contribution, but we are unaware of a case when this has been applied to a wave extrapolation problem, since the cusp term is usually neglected. In the present formulations, with exterior volume sources neglected, it seems likely that contours of $f$ could be given arbitrarily small radii of curvature as long as the other requirements are met.

In practice the source distribution intermediate region will still need to be discretized for computation. This can be formalised by the following intermediate form.

\subsubsection{Stepped function}

For this formulation we set

$$
W(f)=\sum_{i=1}^{N} a_{i} \mathrm{H}\left(f-f_{i}\right)
$$

with $\sum_{i=1}^{N} a_{i}=1$ and $f_{i+1}>f_{i}$ for all $i$. We then have

$$
W^{\prime}(f)=\sum_{i=1}^{N} a_{i} \delta\left(f-f_{i}\right)
$$

This gives a nested set of zero-thickness source layers with contributions weighted according to $a_{i}$, with the exterior volume source distribution between successive layers $i$ and $i+1$ given a stepwise increasing weight between 0 and 1 as $i$ increases (assuming all $a_{i}$ are positive).

The case with all $a_{i}=1 / N$ corresponds to averaging over $N$ FWH surfaces, a strategy shown to be effective when exterior volume sources are neglected by Shur $e t$ al. (2005), Spalart \& Shur (2009) and Mendez et al. (2013). As with the smooth function the optimal distribution of $a_{i}$ values will depend on the statistics of the vorticity. 


\section{Conclusions}

The general formulation of the acoustic analogy (13), or a similar version in terms of a pressure-like variable can be used to obtain different wave extrapolation formulations. These include the classical FWH formulation, a version with a smooth, finite-thickness transition region over which the FWH sources are distributed, and a version with multiple nested extrapolation surfaces. We predict that the second and third will generate less spurious vortex noise than the first when vorticity leaves the interior region. The relative effectiveness and efficiency of this and other proposed solutions would best be tested by numerical examples. We close by remembering Feri Farassat's dedication to the subject of aeroacoustics, and to the community of those who study it.

\section{References}

Avital, E. J., Sandham, N. D. \& Luo, K. H. 1999 Calculation of basic sound radiation of axisymmetric jets by direct numerical simulations. AIAA J. 37, 161-168.

Brentner, K. S. \& Farassat, F. 1998 Analytical comparison of the acoustic analogy and Kirchhoff formulation for moving surfaces. AIAA J. 36, 1379-1386.

Curle, N. 1955 The influence of solid boundaries upon aerodynamic sound. Proc. $R$. Soc. Lond. A 231, 505-514.

Dowling, A. P. \& Ffowcs Williams, J. E. 1983 Sound and Sources of Sound. Chichester: Ellis-Horwood.

Farassat, F. \& Myers, M. K. 1987 Extension of Kirchhoff's formula to radiation from moving surfaces. NASA Technical Memorandum 89149.

Farassat, F. \& Myers, M. K. 1990 An analysis of the quadrupole noise source of high speed rotating blades. In Computational Acoustics Vol. 2 (ed. D. Lee,

A. Cakmak \& R. Vichnevetsky), pp. 227-240, Elsevier. 
Ffowcs Williams, J. E. \& Hawkings, D. L. 1969 Sound generation by turbulence and surfaces in arbitrary motion. Phil. Trans. R. Soc. A 264, 321-342.

di Francescantonio, P. 1997 A new boundary integral formulation for the prediction of sound radiation. J. Sound Vib. 202, 491-509.

Freund, J. B. 2001 Noise sources in a low-Reynolds-number turbulent jet at Mach 0.9. J. Fluid Mech. 134, 1-31.

Ikeda, T., Enomoto, S. \& Yamamoto, K. 2013 On the modification of the Ffowcs Williams-Hawkings integration for jet noise prediction. 19th AIAA/CEAS Aeroacoustics Conference, AIAA-2013-2277.

Lighthill, M. J. 1952 On sound generated aerodynamically. I. General theory. Proc. R. Soc. Lond. A 211, 564-587.

Mendez, S., Shoeybi, M., Lele, S. K. \& Moin, P. 2013 On the use of the Ffowcs Williams-Hawkings equation to predict far-field jet noise from large-eddy simulations. Int. J. Aeroacoustics 12, 1-20.

Morfey, C. L. 1973 Amplification of aerodynamic noise by convected flow inhomogeneities. J. Sound Vib. 31, 391-397.

Morfey, C. L. \& Wright, M. C. M. 2007 Extensions of Lighthill's acoustic analogy with application to computational aeroacoustics. Proc. R. Soc. A 463, 2101-2127.

Morfey, C. L., Powles, C. P. \& Wright, M. C. M. 2011 Green's functions in computational aeroacoustics. Int. J. Aeroacoustics 10, 117-160.

Obrist, D. \& Kleiser, L. 2007 The influence of spatial domain truncation on the prediction of acoustic far-fields. 13th AIAA/CEAS Aeroacoustics Conference, AIAA-2007-3725.

Shur, M. L., Spalart, P. R. \& Strelets, M. K. 2005 Noise prediction for increasingly complex jets. Part I: Methods and tests. Int. J. Aeroacoustics 4, 213-246. 
Spalart, P. R. \& Shur, M. L. 2009 Variants of the Ffowcs Williams-Hawkings equation and their coupling with simulations of hot jets. Int. J. Aeroacoustics $\mathbf{8}$, 477-492.

Wang, M., Lele, S. K. \& Moin, P. 1996 Computation of quadrupole noise using acoustic analogy. AIAA J. 34, 2247-2254. 